\title{
THE SPHERICAL MAXIMAL FUNCTION ON THE FREE TWO-STEP NILPOTENT LIE GROUP
}

\author{
VÉRONIQUE FISCHER*
}

\begin{abstract}
We consider here the free two step nilpotent Lie group, provided with the homogeneous Korányi norm; we prove the $L^{p}$-boundedness of the maximal function corresponding to the homogeneous unit sphere, for some $p$.
\end{abstract}

\section{Introduction}

This article is organized as follows. As introduction, we collect some results and methods of work on singular maximal functions corresponding to averages over dilations of a fixed surface on a nilpotent Lie group. We also present our main result.

Then we define precisely the free two-step nilpotent Lie groups, maximal $L^{p}$-inequality and the square functions used here (Section 2).

Next (Section 3), we prove $L^{p}$-maximal inequalities corresponding to Korányi homogeneous spheres, assuming $L^{2}$-boundedness of our square functions. This hypothesis is proved in Section 5, with the help of the Plancherel formula and spherical functions; these notions are recalled in Section 4.

\subsection{Some maximal functions on nilpotent Lie groups}

On the Euclidean space. Stein proved $L^{p}$-maximal inequalities for Euclidean spheres; that is the supremum of the averages over the spheres centered at a point defines an operator on the space of smooth functions, called "the maximal function corresponding to Euclidean spheres" (or more simply "the spherical maximal function"); and this operator extends to a bounded operator on $L^{p}\left(\mathrm{R}^{n}\right)$. Here $p>n /(n-1), n \geq 3$ (sharp result). Bourgain showed the case $n=2$ [1]; the case $n=1$ is vacuously true.

For the proof in [14], [16], Stein uses:

(i) complex interpolation for the analytic continuation of the family of convolution operator with kernel given by $x \mapsto 2\left(1-|x|^{2}\right)_{+}^{\alpha-1} / \Gamma(\alpha)$,

\footnotetext{
* Author partially supported by the European Commission (IHP Network Harp).
}

Received December 12, 2004; in revised form May 18, 2005. 
(ii) the well known $L^{p}$-boundedness of the standard maximal function (corresponding to the Euclidean balls) [2],

(iii) the $L^{2}$-boundedness of some square functions (with the help of the Euclidean Fourier transform and estimates for special functions).

But Stein gave another proof of the same result in Corollary 3 of [15, XI§3], where he also presented the case of variable manifolds. The method is based there on oscilatory integrals operators and on curvature: in fact, the curvature of the unit Euclidean sphere $S^{n}$ is closely related to the Fourier transform of the probability measure of $\mathrm{S}^{n}[15, \mathrm{XI} \S 1]$.

On Heisenberg groups. M. Cowling offered [3] an alternative approach to Stein's first work on $\mathrm{R}^{n}$; he adapted it on the Heisenberg group $\mathrm{H}^{n}$ for the Korányi norm: he obtained the $L^{p}\left(\mathrm{H}^{n}\right)$-boundedness of the spherical maximal function, $p>(2 n+1) / 2 n$ (sharp result).

Still on the Heisenberg group $\mathrm{H}^{n}=\mathrm{C}^{n} \times \mathrm{R}$, Nevo and Thangavelu considered the case of the complex spheres $\left\{(z, 0) \in \mathrm{C}^{n} \times \mathrm{R}:|z|=r\right\}$, and showed $[19, \S 3.6]$ that the corresponding maximal function is $L^{p}$-bounded for $p>(2 n-1) /(2 n-2)$. He adapted previous works of Nevo on "ergodic" maximal inequalities on some Lie groups (see [8], [11]), where the proofs are based (for the global part) on classical study of maximal functions for semi-groups [13, III§3], and on $L^{2}$-estimates of square functions with spectral calculus (spherical functions).

The optimal range for $p$ is $p>2 n /(2 n-1)$; this was proved recently by Narayanan and Thangavelu [10].

On nilpotent Lie groups. Müller and Seeger obtained [9] also this optimal range for $p$ in a study which concerns more general surfaces (with a non vanishing rotational curvature) in "non-degenerate" two-step nilpotent Lie groups.

In a still unpublished work [12], the maximal functions on stratified group associated to hyper-surfaces with a non vanishing rotational curvature, were also studied by adapting Stein's second proof of the Euclidean case [15, Corollary 3 in XI§3]; it yields to their $L^{p}$-boundedness with $p>n /(n-1)$, where $n \geq 3$ denotes the topological dimension of the group. In particular, it covers the case of the Korányi spheres in groups of Heisenberg type, with a sharp range for $p$, and so includes the result of Cowling [3] on $\mathrm{H}^{n}$.

\subsection{Our main result}

Let $N_{v}$ be the free two step nilpotent Lie group with $v$ generators, provided as a stratified group, with the Korányi norm. Let $\mathscr{A}$ denote the spherical maximal functions. We recall the definitions of these objects in Section 2.

The main result of this paper is the $L^{p}$-boundedness of $\mathscr{A}$ : 
THEOREM 1. Assume $v \geq 4$. The spherical maximal function $\mathscr{A}$ satisfies an $L^{p}\left(N_{v}\right)$-inequality where $2 h /(2 h-1)<p \leq \infty$ and $h$ is the largest integer such that $h<v(v-1) / 4-1$.

This result is new, but probably not sharp for the range of $p$. The group $N_{v}$ does not fulfill the non-degeneracy condition of [9], and the surface is not of the type studied there neither. It is not covered by the study [12] because the curvature of the unit homogeneous sphere in $N_{v}$ vanishes "at the equator", $v \geq 3$.

Our proof follows the first method of the Euclidean case [14], i.e. the points (i), (ii), (iii); but the Euclidean norm is replaced by the Korányi norm and for $L^{2}$-estimates of square functions (i.e. for (iii)), we use the Plancherel formula and the bounded spherical functions. This method for groups of Heisenberg type (and always Korányi homogeneous spheres) gives a result included in [12] (see the French $\mathrm{PhD}$ thesis of the author [5]).

The rest of this paper is organized as follows. We start by setting definitions and notations for the group, the spherical maximal function and square functions denoted $S^{j}$ (Section 2). We give then the proof of our main theorem (Section 3), provided that some square functions $S^{j}$ satisfy $L^{2}$-estimates. Next (Section 5) we prove this hypothesis, using the Plancherel formula and spherical functions (notions recalled in Section 4).

\section{Definitions and Notations}

Here we set definitions and notations for the group, the spherical maximal function and the square functions that we study.

In this paper, we assume $v$ is an integer such that $v \geq 2$.

\subsection{Free two-step nilpotent Lie algebras and groups}

Let $\mathcal{N}_{v}$ be the (unique up to isomorphism) free two-step nilpotent Lie algebra with $v$ generators; the definition using the universal property can be found in [7, Chapter V §4]. Roughly speaking, $\mathcal{N}_{v}$ is a two-step nilpotent Lie algebra with $v$ generators $X_{1}, \ldots, X_{v}$, such that the vectors $X_{1}, \ldots, X_{v}$ and $X_{i, j}=\left[X_{i}, X_{j}\right]$, $i<j$, form a basis of the vector space $\mathcal{N}_{v}$.

We denote by $\mathscr{V}$ and $\mathscr{Z}$, the vector spaces generated by the families of vectors $X_{1}, \ldots, X_{v}$ and $X_{i, j}:=\left[X_{i}, X_{j}\right], 1 \leq i<j \leq v$ respectively; these families become the canonical bases of $\mathscr{V}$ and $\mathscr{Z}$. Thus $\mathcal{N}_{v}=\mathscr{V} \oplus \mathscr{Z}$ is a stratified algebra (see [6]), and $\mathscr{Z}$ is the center of the Lie algebra $\mathscr{N}_{v}$. Let $z=v(v-1) / 2$ be the dimension of $\mathscr{Z}$ and $Q=v+2 z=v^{2}$ be the homogeneous dimension. We write $v=2 v^{\prime}$ or $2 v^{\prime}+1$.

The connected simply connected nilpotent Lie group which corresponds to $\mathscr{N}_{v}$ is called the free two-step nilpotent Lie group and is denoted $N_{v}$. We 
denote by exp $: \mathscr{N}_{v} \rightarrow N_{v}$ the exponential map. In this paper, we use the notations $X+A \in \mathcal{N}, \exp (X+A) \in N$ when $X \in \mathscr{V}, A \in \mathscr{Z}$. For an element $n=\exp (X+A) \in N$, we define the dilations: $r . n=\exp \left(r X+r^{2} A\right)$, $r>0$, and the (homogeneous) Korányi norm: $|n|=\left(|X|^{4}+|A|^{2}\right)^{\frac{1}{4}}$ (see [6]).

\subsection{Some measures}

The canonical bases of $\mathscr{V}$ and $\mathscr{Z}$ induce Lebesgue measures on $\mathscr{V}$ and $\mathscr{Z}$ respectively, thus on $\mathcal{N}$, and a Haar measure $d n$ on $N_{v}$. In this paper, the $L^{p}$ spaces over $N_{v}$ and the measure $|E|$ for a Borel set $E \subset N_{v}$ are related to the Haar measure $d n$.

We denote $\mu$ the unique Radon measure on the unit homogeneous sphere $S_{1}:=\left\{n \in N_{v},|n|=1\right\}$ such that for each integrable function $f$ on $N_{v}$, we have [6, prop.1.15]:

$$
\int_{N} f(n) d n=\int_{r=0}^{\infty} \int_{S_{1}} f(r . n) d \mu(n) r^{Q-1} d r .
$$

By uniqueness of (1), and with suitable changes of variables, we easily obtain:

$$
\text { (2) } \begin{aligned}
& \int_{S_{1}} f(n) d \mu(n) \\
= & 2 \int_{0}^{1} \int_{\mathbf{S}^{v}} \int_{\mathbf{S}^{z}} f\left(\exp t X+\sqrt{\left(1-t^{4}\right)} Z\right) d \sigma_{z}(Z) d \sigma_{v}(X) t^{v-1}\left(1-t^{4}\right)^{\frac{z-2}{2}} d t .
\end{aligned}
$$

(where $\sigma_{n}$ denotes the probability measure on the Euclidean sphere $\mathbf{S}^{n} \subset \mathbf{R}^{n}$ ). It is still valid for any two-step stratified group.

\subsection{Maximal $L^{p}$-inequality}

Here, we recall the definition of the maximal function associated to an operator over $N_{v}$, in particular of the spherical maximal function $\mathscr{A}$, and of the standard maximal function $\mathscr{M}$; this is still valid for any stratified group.

Let us start with the definitions of dilations for subsets, distributions and operators over $N_{v}$. Let $r>0$. The $r$-dilation of $E \subset N_{v}$ is $r . E=\{r . n, n \in E\}$, and if $E$ is a measurable set, we have $|r . E|=r^{Q}|E|$. The dilation $F_{r}$ of a distribution $F$ on $N_{v}$, is given for a test function $f$ on $N_{v}$ by $\left\langle F_{r}, f\right\rangle=$ $\langle F, f(r)$.$\rangle where f(r):. n \mapsto f(r . n)$. The $r$-dilation $T_{r}$ of an operator $T$ on a dilation invariant space of function on $N_{v}$ is given for a function $f$ by: $T_{r} . f=$ $(T f(r)).\left(r^{-1}\right.$.). For a convolution operator with an integrable function or a measure, the $r$-dilated operator coincides with the convolution operator with the corresponding $r$-dilated function or measure.

Let $T$ be an operator on a dilation invariant space of function on $N_{v}$. We define the maximal function associated to $T: \mathscr{T}: f \mapsto \sup _{r>0}\left|T_{r} . f\right|$. We say 
that $\mathscr{T}$ satisfies an $L^{p}$-inequality (or $T$ satisfies an $L^{p}$ maximal inequality, or $\mathscr{T}$ is $L^{p}$-bounded) if there exists a constant $C=C(T, v, p)>0$ such that for all smooth compactly supported functions $f$ on $N_{v}$ (or equivalently the class $L^{p}\left(N_{v}\right)$, the Schwartz class $\left.\mathscr{S}\left(N_{v}\right) \ldots\right)$, we have: $\|\mathscr{T} . f\|_{L^{p}} \leq C\|f\|_{L^{p}}$.

The maximal spherical function $\mathscr{A}$ is the maximal function associated to the convolution operator with the measure $\mu$ (see (1)). The aim of this paper is to establish $L^{p}$-inequalities for $\mathscr{A}$.

Let $\mathcal{M}$ denote the standard maximal function, i.e. the maximal function associated to the characteristic function of the unit homogeneous ball $B_{1}:=$ $\left\{n \in N_{v} ;|n| \leq 1\right\}$. During the proof of our main result, we will use the well known $L^{p}$-inequalities $(1<p \leq \infty)$, satisfied by the standard maximal function $\mathscr{M}$ [2]. We will also need the following corollary:

Corollary 2. Let $m: \mathbf{R}^{+} \rightarrow \mathbf{R}^{+}$be a decreasing function and $F:$ $N \rightarrow \mathrm{R}^{+}$be defined by $F(n)=m(|n|), n \in N_{v}$. Suppose $F \in L^{1}$. The maximal function associated to the convolution operator with $F$ satisfies $L^{p}$ inequalities, for $1<p \leq \infty$.

\subsection{Square functions}

For $j=1,2, \ldots$, we define the following operator, called the square function $S^{j}$, by:

$$
S^{j}(f)(n):=\sqrt{\int_{s=0}^{\infty}\left|\partial_{s}^{j}\left(f * \mu_{s}\right)(n)\right|^{2} s^{2 j-1} d s,} \quad f \in \mathscr{S}\left(N_{v}\right), \quad n \in N_{v} .
$$

We will have to show that for some $j$, the square functions $S^{j}$ satisfy $L^{2}$ estimates:

$$
\text { i.e } \exists C=C(v, j)>0 \quad \forall f \in \mathscr{S}\left(N_{v}\right) \quad\left\|S^{j} \cdot f\right\|_{L^{2}} \leq C\|f\|_{L^{2}} .
$$

In Section 5, using the Plancherel formula and spherical functions, we will obtain:

Theorem 3. We assume $v \geq 4$. The square functions $S^{j}, j=1, \ldots, h$, satisfy $L^{2}$-estimates, where $h \in \mathrm{N}, 1 \leq h<(z-2) / 2$.

\section{Proof of $L^{p}$-maximal estimates for $\mathscr{A}$}

Here we will prove that the $L^{2}$-boundedness of some square functions $S^{j}$ implies the $L^{p}$-boundedness of $\mathscr{A}$, for some $p$. We summarize this fact in the following Theorem:

THEOREM 4. If the square functions $S^{j}$ satisfy $L^{2}$-estimates, then the spherical maximal function $\mathscr{A}$ satisfies $L^{p}$-estimates, where: 
a) $j=1$ and $2 \leq p \leq \infty$ if $v \geq 2$,

b) $1<j \leq h<(Q-2) / 2$ and $2 h /(2 h-1)<p \leq \infty$ if $v>2$.

This proof extends naturally to any stratified Lie groups. Furthermore Theorems 3 and 4 imply Theorem 1 . In this section, we denote $N_{v}=N$.

\subsection{Proof of the $L^{p}$-case, $p \geq 2$}

Here we prove Theorem 4.a): we assume that the square function $S^{1}$ is $L^{2}$ bounded and we prove the $L^{p}$-boundedness of $\mathscr{A}, p \geq 2$.

We follow the Euclidean process [15, XI§1]. For $f \in \mathscr{S}(N)$, we have:

$$
\begin{aligned}
\mu_{t} * f(n) & =\frac{1}{t^{Q}} \int_{0}^{t} \partial_{s}\left(s^{Q} \mu_{s} * f(n)\right) d s \\
& =\frac{1}{t^{Q}} \int_{0}^{t} Q s^{Q-1} \mu_{s} * f(n) d s+\frac{1}{t^{Q}} \int_{0}^{t} s^{Q} \partial_{s}\left(\mu_{s} * f(n)\right) d s .
\end{aligned}
$$

We compute $t^{Q}=|B(n, t)| /\left|B_{1}\right|$, where $B(n, r)=\left\{n^{\prime} \in N,\left|n n^{\prime-1}\right| \leq r\right\}$ is the homogeneous ball with radius $r$, centered at $n$, and with change in polar coordinates (1):

$$
\frac{1}{t^{Q}} \int_{0}^{t} Q s^{Q-1} \mu_{s} * f(n) d s=Q \frac{\left|B_{1}\right|}{|B(n, t)|} \int_{B(n, t)} f\left(n^{\prime \prime}\right) d n^{\prime \prime}
$$

Because of Hölder's inequality, the last expression in (3), is majorized by $(2 Q)^{-1 / 2} S^{1} \cdot f(n)$. Finally, we gather:

$$
\left|\mu_{t} * . f(n)\right| \leq Q \frac{\left|B_{1}\right|}{|B(n, t)|} \int_{B(n, t)} f\left(n^{\prime \prime}\right) d n^{\prime \prime}+\frac{1}{\sqrt{2 Q}} S^{1} \cdot f(n) .
$$

Taking the supremum over $t$, we have the following pointwise estimate:

$$
\forall f \in \mathscr{S}(N) \quad \mathscr{A} \cdot f \leq Q\left|B_{1}\right| \mathcal{M} \cdot f+\frac{1}{\sqrt{2 Q}} S^{1} \cdot f .
$$

The standard maximal function $\mathscr{M}$ satisfies $L^{p}$-inequalities, in particular for $p=2$ [2]. As the square function $S^{1}$ is assumed to be $L^{2}$-controlled, the inequality (4) leads to an $L^{2}$-inequality for $\mathscr{A}$. But $\mathscr{A}$ satisfies also an $L^{\infty}$ inequality (because the measure $\mu$ is finite). By classical real interpolation, we obtain the $L^{p}$-boundedness of $\mathscr{A}$, for $p \geq 2$. Theorem 4.a) is thus proved. 


\subsection{Overview of the proof of the $L^{p}$-cases, $p<2$}

Here we prove Theorem 4.b). We start with a choice of an analytic family of operators $A^{\alpha}$, the same as in the Euclidean case [14], [16], see (i) of Subsection 1.1. $A^{0}$ coincides with the convolution operator with kernel $\mu$. Provided that the square functions $S^{j}, 1 \leq j \leq h$, satisfy $L^{2}$-estimates, we prove that $A^{\alpha}$ satisfies $L^{p}$-maximal inequalities, for $\Re \alpha \geq 1$ with $1<p \leq \infty$ (Subsection 3.4), and for $\Re \alpha>-h+1$ with $p=2$ (Subsection 3.5). To obtain these maximal inequalities, we will use Corollary 2 , and the following estimate "uniformly in $y \in \mathrm{R}$, locally in $x>0$ " for the $\Gamma$ function [20, page 151]:

$$
\forall[a, b] \subset[0, \infty[\quad \exists C>0 \quad \forall x \in[a, b] \quad \forall y \in \mathbf{R}
$$

$$
C^{-1} \leq \frac{e^{-\frac{\pi}{2} y}|y|^{x-\frac{1}{2}}}{|\Gamma(x+i y)|} \leq C .
$$

From these maximal inequalities, with interpolation (Subsection 3.6), we will deduce Theorem 4.b).

\subsection{Analytic family of operators}

Let define (as in the Euclidean case, see [14] or Subsection 1.1) for $\alpha \in$ $\mathrm{C} \backslash(-\mathrm{N})$ :

$$
m^{\alpha}(r)=\frac{2\left(1-|r|^{2}\right)_{+}^{\alpha-1}}{\Gamma(\alpha)}, \quad r \geq 0,
$$

and

$$
\mu^{\alpha}(n)=m^{\alpha}(|n|), \quad n \in N .
$$

For $\Re \alpha>0$, the function $\mu^{\alpha}$ is radial and integrable; we denote by $A^{\alpha}$ the convolution operator with kernel $\mu^{\alpha}$; it is a $L^{p}$-bounded operator for $1<p \leq$ $\infty$.

Let $j, h \in \mathrm{N}$ such that $0 \leq j \leq h$ and $1 \leq h>Q / 2$; we define also the operator $B_{h, j}^{\alpha}$ for $f \in \mathscr{S}(N)$, and $n \in N$, by:

$$
B_{h, j}^{\alpha}(f)(n)=\int_{r=0}^{1} m^{\alpha+h}(r) r^{Q-1-2 h+j} \partial_{r}^{j}\left(f * \mu_{r}\right)(n) d r .
$$

Proposition 5. The family of operators $\left\{A^{\alpha}, \Re \alpha>0\right\}$ is analytic, and admits an analytic continuation over $\{\Re \alpha>1-(Q-2) / 2\}$ as operators on $\mathscr{S}(N)$. The operator $A^{0}$ coincides with the convolution operator with kernel $\mu$. For $\Re \alpha>1-h$ with $h<(Q-2) / 2, A^{\alpha}$ can be written as linear combination of the operators $B_{h, j}^{\alpha}, 0 \leq j \leq h$. 
Proof of Proposition 5. With the change in polar coordinates (1):

$$
\begin{aligned}
A^{\alpha} \cdot f= & \int_{r=0}^{1} \frac{2}{\Gamma(\alpha)}\left(1-r^{2}\right)^{\alpha-1}\left(f * \mu_{r}\right) r^{Q-1} d r \\
= & {\left[\frac{2}{\alpha \Gamma(\alpha)}\left(1-r^{2}\right)^{\alpha} \frac{1}{-2 r}\left(f * \mu_{r}\right) r^{Q-1}\right]_{r=0}^{1} } \\
& \quad-\int_{r=0}^{1} \frac{2}{\alpha \Gamma(\alpha)}\left(1-r^{2}\right)^{\alpha} \partial_{r}\left(\frac{1}{-2 r}\left(f * \mu_{r}\right) r^{Q-1}\right) d r
\end{aligned}
$$

after integrating by parts. The endpoint terms equal zero, and $\alpha \Gamma(\alpha)=\Gamma(\alpha+$ 1); we easily obtain on one hand for $\alpha=0: A^{0} \cdot f=f * \mu$, and on the other hand after expanding the derivative: $A^{\alpha}=-1 / 2\left(B_{1,1}+Q B_{1,0}\right)$.

Recursively, using $h$ integrations by parts, we easily compute that the operator $A^{\alpha}$ coincides with a linear combination of the operators $B_{h, j}^{\alpha}$, provided that the endpoint terms equal zero, so as long as $h<(Q-2) / 2$ and $\Re \alpha>1-h$.

Now we establish some $L^{p}$-maximal inequalities on $A^{\alpha}$. We denote by $\mathscr{A}^{\alpha}$ the maximal function associated to $A^{\alpha}$ (see Subsection 2.3).

\section{4. $L^{p}$-maximal inequalities for $\Re \alpha \geq 1$}

In this subsection, using Corollary 2 and estimation (5), we prove:

Proposition 6. For $1<p \leq \infty$, we have:

$$
\begin{gathered}
\forall b \geq 1 \quad \forall x \in[1, b] \quad \exists C>0 \quad \forall y \in \mathrm{R} \quad \forall f \in \mathscr{S}(N), \\
\left\|\mathscr{A}^{x+i y} \cdot f\right\|_{L^{p}} \leq C e^{2|y|}\|f\|_{L^{p}} .
\end{gathered}
$$

Proof of Proposition 6. For $x>0$, and $f \in \mathscr{S}(N)$, we have:

$$
\left|A^{x+i y} \cdot f\right| \leq|f| *\left|\mu^{x+i y}\right|=\left|\frac{\Gamma(x)}{\Gamma(x+i y)}\right||f| * \mu^{x} \leq C e^{2|y|} A^{x} \cdot|f| .
$$

uniformly in $y \in \mathrm{R}$, locally for $x>0$, because of (5). It yields to $\mathscr{A}^{x+i y} \cdot f \leq$ $C e^{2|y|} \mathscr{A}^{x} \cdot|f|$. Now, For $x \geq 1, \mu^{x}$ satisfies the hypothesis of Corollary 2, and the maximal function $\mathscr{A}^{x}$ is $L^{p}$-bounded, $1<p \leq \infty$.

\section{5. $L^{p}$-maximal inequalities for $\Re \alpha<0$}

In this subsection, we fix $h=1,2, \ldots$ and we prove:

Proposition 7. If the square functions $S^{j}, j=1, \ldots, h$ satisfy $L^{2}$ estimates, then for all segments $[a, b] \subset]-h+1, \infty[$, there exists a constant 
C $>0$ such that:

$$
\forall x \in[a, b] \quad \forall y \in \mathbf{R} \quad \forall f \in \mathscr{S}(N), \quad\left\|\mathscr{A}^{x+i y} \cdot f\right\|_{L^{2}} \leq C e^{2|y|}\|f\|_{L^{2}} .
$$

This proposition is implied by the following lemma, which states estimates for the maximal function $\mathscr{B}_{h, j}^{\alpha}$ associated (see Subsection 2.3) to $B_{h, j}^{\alpha}$ :

Lemma 8. For all segments $[a, b] \subset]-h+1, \infty[$, there exists a constant $C>0$ such that for all $x \in[a, b]$ and for all $y \in \mathrm{R}$, we have:

$$
\begin{aligned}
& \forall f \in L^{2} \quad\left\|\mathscr{B}_{h, 0}^{x+i y} \cdot f\right\|_{L^{2}} \leq C e^{2|y|}\|f\|_{L^{2}}, \\
& \forall f \in \mathscr{S}(N) \quad\left|\mathscr{B}_{h, j}^{x+i y} . f\right| \leq C e^{2|y|}\left|S^{j} \cdot f\right| .
\end{aligned}
$$

The proof of Lemma 8 is based on Corollary 2, known formulae for $\Gamma$ and estimation (5).

Proof OF Lemma 8. Because of the change in polar coordinates (1), we compute that $B_{h, 0}^{\alpha}$ is the convolution operator with kernel given by: $n \mapsto$ $\mu^{\alpha+h}(n)|n|^{-2 h}, n \in N$. Then we proceed as in the proof of Proposition 6 to obtain inequality (6).

Let us prove inequality (7). For $f \in \mathscr{S}(N)$, we have:

$$
\left(B_{h, j}^{\alpha}\right)_{t} \cdot f=\int_{r=0}^{1} m^{\alpha+h}(r) r^{Q-1-2 h+j} t^{j}\left[\partial_{r^{\prime}}^{j} f * \mu_{r^{\prime}}\right]_{\mid r^{\prime}=r t} d r .
$$

Thus because of Hölder's inequality, $\left(\mathscr{B}_{h, j}^{\alpha} . f\right)^{2}$ is majorized by the product of

$$
\begin{aligned}
& \sup _{t>0} \int_{r=0}^{1}\left|r^{j-\frac{1}{2}} t^{j}\left[\partial_{r^{\prime}}^{j} f * \mu_{r^{\prime}}\right]_{\mid r^{\prime}=r t}\right|^{2} d r \leq \int_{r^{\prime}=0}^{\infty}\left|\partial_{r^{\prime}}^{j} f * \mu_{r^{\prime}}\right|^{2} r^{\prime 2 j-1} d r^{\prime} \\
& =\left(S^{j}(f)\right)^{2},
\end{aligned}
$$

(with the help of change of variable $r^{\prime}=r t$ ) and the integral:

$$
\int_{r=0}^{1}\left|m^{\alpha+h}(r) r^{Q-1-2 h-\frac{1}{2}}\right|^{2} d r \leq \int_{r=0}^{1} \frac{\left(1-r^{2}\right)^{2(x+h-1)}}{|\Gamma(\alpha+h)|^{2}} r^{2 Q-1-4 h} d r .
$$

This last integral equals with the change of variable $r^{\prime}=r^{2}$, then with known formulae concerning the $\Gamma$ function $[17, \S 1.7$ (4) and (5)]:

$$
\int_{r^{\prime}=0}^{1} \frac{\left(1-r^{\prime}\right)^{2(x+h-1)}}{|\Gamma(\alpha+h)|^{2}} r^{\prime} Q-1-2 h \frac{d r^{\prime}}{2}=\frac{1}{2} \frac{\Gamma(2 x+2 h-1) \Gamma(Q-2 h)}{|\Gamma(\alpha+h)|^{2} \Gamma(2 x+Q)} .
$$


Because of estimation (5), this term is bounded up to a constant, locally in $x>-h+1$, uniformly in $y \in \mathrm{R}$, by $e^{2|y|}$. We obtain inequality (7).

This ends the proofs of Lemma 8 thus of Proposition 7.

\subsection{Interpolation}

The maximal function $\mathscr{A}=2 \mathscr{A}^{0}$ (Proposition 5) satisfies an $L^{\infty}$-inequality because the measure $\mu$ is finite. We have proved in Propositions 7 and 6, that the family $\mathscr{A}^{\alpha}$ satisfies $L^{p}$-maximal inequalities. Using linearisation and interpolation of an analytic family, we obtain that the maximal function $\mathscr{A}^{0}$ satisfies $L^{p}$-inequalities for $(2 h) /(2 h-1)<p \leq \infty$ as long as the square functions $S^{j}, 1 \leq j \leq h$ satisfy $L^{2}$-estimates and $1<h<(Q-2) / 2$ (so $v \geq 3$ ).

We have proved Theorem 4.b). This achieves the proof of Theorem 4, and thus of our main theorem, provided that we show Theorem 3. The last section (Section 5) is devoted to this, but we start (Section 4) by recalling our tools: the Plancherel formula and bounded spherical functions.

\section{The Bounded Spherical Functions and the Plancherel Formula}

Here we present the tools that we will need in the proof of Theorem 3: the Plancherel formula and bounded spherical functions on $N_{v}$. For this results, we refer the interested reader to [5].

\subsection{Action of $O(v)$}

With the canonical basis, the vector space $\mathscr{Z}$ can be identified with the vector space of skew-symmetric $v \times v$-matrices $M_{v}^{s s}$.

The group $O(v)$ acts on $\mathscr{V}_{v} \sim \mathrm{R}^{v}$ and $\mathscr{Z}_{v} \sim M_{v}^{s s}$ by auto-morphisms:

$$
\left\{\begin{array} { r l } 
{ O ( v ) \times \mathscr { V } _ { v } } & { \longrightarrow \mathscr { V } _ { v } } \\
{ ( k , X ) } & { \longmapsto k . X }
\end{array} \quad \text { and } \quad \left\{\begin{array}{rl}
O(v) \times \mathscr{Z}_{v} & \longrightarrow \mathscr{Z}_{v} \\
(k, A) & \longmapsto k \cdot A=k A k^{-1} .
\end{array}\right.\right.
$$

We get easily $k .\left[X, X^{\prime}\right]=\left[k . X, k . X^{\prime}\right], k \in O(v), X, X^{\prime} \in \mathscr{V}_{v}$. So, $O(v)$ acts (by auto-morphisms) on the Lie algebra $\mathscr{N}_{v}$, then on the group $N_{v}$.

A distribution or a left invariant differential operator on $N_{v}$ is said to be radial, if it is $O(v)$-invariant.

\subsection{Special functions}

First, let set the notations for "our" Hermite, Laguerre and Bessel functions. We denote:

- $h_{l}, l \in \mathrm{N}$ the Hermite-Weber functions on $\mathrm{R}$ given by [17, §5.5]:

$$
h_{l}(x)=\left(2^{l} l ! \sqrt{\pi}\right)^{-\frac{l}{2}} e^{-\frac{x^{2}}{2}} H_{l}(x) \text { where } H_{l}(s)=(-1)^{l} e^{s^{2}}(d / d s)^{l} e^{-s^{2}} \text {, }
$$


- $\mathscr{L}_{n}$, the Laguerre function given by $\mathscr{L}_{n}(x)=L_{n}^{0}(x) e^{-\frac{x}{2}}$ where $L_{n}^{0}$ is the Laguerre polynomial of type 0 and degree $n[17, \S 5.1]$,

- $\mathscr{J}_{\alpha}$ the reduced Bessel function: $\mathscr{J}_{\alpha}(z):=\Gamma(\alpha+1)(z / 2)^{-\alpha} J_{\alpha}(z)$, where $J_{\alpha}$ is the Bessel function of type $\alpha>0$ [17, $\left.\S 1.71\right]$, [4, ch. II, I.1].

Now, we can give some spherical (or equivalently of positive type) functions of $\left(N_{v}, O(v)\right)$. We denote:

- $d k$ the Haar probability measure of $O(v)$,

- for $\Lambda=\left(\lambda_{1}, \ldots, \lambda_{v^{\prime}}\right) \in \mathrm{R}^{v^{\prime}}, D_{2}(\Lambda)$ an element of $\mathscr{Z}_{v}^{*}$ identified with the skew-symmetric matrix:

$$
D_{2}(\Lambda):=\left[\begin{array}{cccc}
\lambda_{1} J & & & \\
0 & \ddots & 0 & \\
& & \lambda_{v^{\prime}} J & \\
& & & (0)
\end{array}\right] \text { where } J:=\left[\begin{array}{rr}
0 & 1 \\
-1 & 0
\end{array}\right] .
$$

((0) means that a zero appears only in the case $v=2 v^{\prime}+1$.)

- $X_{1}^{*}, \ldots, X_{v}^{*}$ the dual base of $X_{1}, \ldots, X_{v}$,

- $\operatorname{pr}_{j}$ the orthogonal projection on $\mathrm{R} X_{2 j-1} \oplus \mathrm{R} X_{2 j}$, for $j=1, \ldots, v^{\prime}$,

- $\mathscr{L}$ the set of $\Lambda=\left(\lambda_{1}, \ldots, \lambda_{v^{\prime}}\right) \in \mathrm{R}^{v^{\prime}}$ such that $\lambda_{1}>\cdots>\lambda_{v^{\prime}}>0$,

- $\mathcal{M}$ the set of $(r, \Lambda)$ with $\Lambda \in \mathscr{L}$ and $r>0$ if $v=2 v^{\prime}+1$ or $r=0$ if $v=2 v^{\prime}$

- $\mathscr{P}$ the set of $(r, \Lambda, l)$ with $(r, \Lambda) \in \mathcal{M}$ and $l \in \mathbf{N}^{v^{\prime}}$.

For $(r, \Lambda, l) \in \mathscr{P}$, we define the function $\phi^{r, \Lambda, l}$ by:

$$
\phi^{r, \Lambda, l}(n)=\int_{O(v)} \Theta^{r, \Lambda, l, \epsilon}(k . n) d k, \quad n \in N_{v},
$$

where $\Theta^{r, \Lambda, l}$ is given by:

$$
\Theta^{r, \Lambda, l}(\exp (X+A))=e^{i\left\langle r X_{v}^{*}, X\right\rangle} e^{i\left\langle D_{2}(\Lambda), A\right\rangle} \prod_{j=1}^{v^{\prime}} \mathscr{L}_{l_{j}}\left(\frac{\lambda_{j}}{2}\left|\operatorname{pr}_{j}(X)\right|^{2}\right) .
$$

The function $\phi^{r, \Lambda, l}$ is a spherical function of $\left(N_{v}, O(v)\right)$. In the rest of this article, we identify a function $\phi^{r, \Lambda, l}$, with its parameter $(r, \Lambda, l) \in \mathscr{P}$. 


\subsection{The Plancherel Formula}

Let $(r, \Lambda) \in \mathscr{M}$. Let define the representation $\left(L^{2}\left(\mathrm{R}^{v^{\prime}}\right), \Pi_{r, \Lambda}\right) \in \hat{N}_{v}$ by:

$$
\begin{aligned}
\prod_{r, \Lambda}(n) \cdot f(y)=\exp \left(i \sum_{j=1}^{v^{\prime}} \frac{\lambda_{j}}{2} x_{2 j} x_{2 j-1}+\sqrt{\lambda_{j}} x_{2 j} y_{j}\right) e^{i r x_{v}+i\left\langle D_{2}(\Lambda), A\right\rangle} & \\
& f\left(y_{1}+\sqrt{\lambda_{1}} x_{1}, \ldots, y_{v^{\prime}}+\sqrt{\lambda_{v^{\prime}}} x_{2 v^{\prime}-1}\right),
\end{aligned}
$$

where $f \in L^{2}\left(\mathbf{R}^{v^{\prime}}\right),\left(y_{1}, \ldots, y_{v^{\prime}}\right) \in \mathbf{R}^{v^{\prime}}, n=\exp (X+A) \in N_{v}$ with $X=$ $\sum_{j=1}^{v} x_{j} X_{j}$.

The group $O(v)$ acts on $N_{v}$ thus also on $\hat{N}_{v}$. In particular, we denote:

$$
k . \prod_{r, \Lambda}(n)=\prod_{r, \Lambda}\left(k^{-1} . n\right), \quad k \in O(v), \quad n \in N_{v} .
$$

We denote $\eta$ the measure on $\mathscr{L}$ given by:

$$
d \eta(\Lambda)= \begin{cases}\prod_{i=1}^{v^{\prime}} \lambda_{i} \prod_{j<k}\left(\lambda_{j}^{2}-\lambda_{k}^{2}\right)^{2} d \lambda_{1} \ldots d \lambda_{v^{\prime}} & \text { if } v=2 v^{\prime}, \\ \prod_{i=1}^{v^{\prime}} \lambda_{i}^{3} \prod_{j<k}\left(\lambda_{j}^{2}-\lambda_{k}^{2}\right)^{2} d \lambda_{1} \ldots d \lambda_{v^{\prime}} & \text { if } v=2 v^{\prime}+1,\end{cases}
$$

and by $\tau$ the Lebesgue measure on $\mathrm{R}^{*+}$ if $v=2 v^{\prime}+1$, and the Dirac measure in 0 if $v=2 v^{\prime}$. Let $m$ be the measure on $\mathscr{M} \times O(v)$ given as the tensor product of $\tau, \eta$ and $d k$, up to a normalizing constant so that we have the Plancherel formula:

Theorem 9. $m$ is the Plancherel measure of $N_{v}$ :

$$
\forall f \in \mathscr{S}\left(N_{v}\right) \quad\|f\|_{L^{2}}^{2}=\int\left\|k \cdot \Pi_{r, \Lambda}(f)\right\|_{H S}^{2} d m(r, \Lambda, k),
$$

where $\|\cdot\|_{H S}$ denotes the norm of an Hilbert-Schmidt operator.

For $l=\left(l_{1}, \ldots, l_{v^{\prime}}\right) \in \mathbf{N}^{v^{\prime}}$, let $\zeta_{l} \in L^{2}\left(\mathbf{R}^{v^{\prime}}\right)$ be given by: $\zeta_{l}\left(y_{1}, \ldots, y_{v^{\prime}}\right)=$ $\Pi_{j=1}^{v^{\prime}} h_{l_{j}}\left(y_{j}\right)$. The vectors $\zeta_{l}, l \in \mathbf{N}^{v^{\prime}}$, form an orthonormal basis of $L^{2}\left(\mathbf{R}^{v^{\prime}}\right)$. If $f$ is radial, the Hilbert-Schmidt norm of $\Pi(f)$, can be computed with this basis, in term of spherical functions:

Proposition 10. Let $f$ be a radial integrable function (or a radial finite measure) on $N_{v}$. We have:

$$
\Pi_{r, \Lambda}(f) . \zeta_{l}=\left\langle f, \phi^{r, \Lambda, l}\right\rangle \zeta_{l} .
$$




\section{Square functions}

This section is devoted to the proof of Theorem 3. Actually, we will prove (Subsection 5.1) that the $L^{2}$-norm of square functions $S^{j}, j=1,2, \ldots$, is bounded by the $L^{\infty}$-norm of:

$$
\hat{S}^{j}(\phi):=\sqrt{\int_{s=0}^{\infty}\left|\partial_{s}^{j}\left\langle\mu_{s}, \phi\right\rangle\right|^{2} s^{2 j-1} d s}, \quad \phi \in \mathscr{P} .
$$

In this section, $\|$.$\| denotes the L^{2}\left(N_{v}\right)$-norm.

Proposition 11. We have for $f \in \mathscr{S}\left(N_{v}\right)$ and $j \geq 1$ :

$$
\left\|S^{j}(f)\right\| \leq \sup _{\phi \in \mathscr{P}} \hat{S}^{j}(\phi)\|f\| .
$$

Next (Subsection 5.2), we obtain the following boundedness for $\hat{S}^{j}$ :

Proposition 12. Let $h$ be an integer such that $1 \leq h<(z-2) / 2$ (only for $v \geq 4)$. We have:

$$
\exists C>0 \quad \forall \phi \in \mathscr{P} \quad \hat{S}^{h}(\phi) \leq C .
$$

For the last proposition, we will use technical lemmas, which will be proved in Subsection 5.3.

Propositions 11 and 12 imply Theorem 3.

\section{1. $S^{j}$ and $\hat{S}^{j}$}

Here, we prove Proposition 11. Let $f \in \mathscr{S}\left(N_{v}\right)$. With the Plancherel formula of Section 4, we will prove in this subsection that we have:

$$
\left\|S^{j}(f)\right\|^{2}=\int \sum_{l \in \mathbb{N}^{v^{\prime}}}\left|\hat{S}^{j}\left(\phi^{r, \Lambda, l}\right)\right|^{2}\left|k \cdot \Pi_{r, \Lambda}(f) \cdot \zeta_{l}\right|^{2} d m(r, k, \Lambda) .
$$

Because of the Plancherel formula (see Theorem 9), this equality implies:

$$
\begin{aligned}
\left\|S^{j}(f)\right\|^{2} & \leq \sup _{\phi \in \mathscr{P}}\left|\hat{S}^{j}(\phi)\right|^{2} \int \sum_{l \in \mathbb{N}^{\prime}}\left|k \cdot \Pi_{r, \Lambda}(f) \cdot \zeta_{l}\right|^{2} d m(r, k, \Lambda) \\
& =\sup _{\phi \in \mathscr{P}}\left|\hat{S}^{j}(\phi)\right|^{2}\|f\|^{2} .
\end{aligned}
$$

Thus Proposition 11 is proved, if equality (8) is satisfied. 
Let us establish equality (8). Fubini's equality and the Plancherel formula (Theorem 9) for $\partial_{s}^{j}\left(f * \mu_{s}\right) \in \mathscr{S}(N)$ yield to:

(9) $\left\|S^{j}(f)\right\|^{2}=\int_{s=0}^{\infty} \int \sum_{l \in \mathbb{N}^{v^{\prime}}}\left|k \cdot \Pi_{r, \Lambda}\left(\partial_{s}^{j}\left(f * \mu_{s}\right)\right) \cdot \zeta_{l}\right|^{2} d m(r, \Lambda, k) s^{2 j-1} d s$.

Because $\mu_{s}$ is a radial probability measure, $k . \Pi_{r, \Lambda}\left(\mu_{s}\right)$ equals $\Pi_{r, \Lambda}\left(\mu_{s}\right)$, and we have (Proposition 10): $\Pi_{r, \Lambda}\left(\mu_{s}\right) \cdot \zeta_{l}=\left\langle\mu_{s}, \phi^{r, \Lambda, l}\right\rangle \zeta_{l}$. Then we deduce:

$$
\begin{aligned}
k . \Pi_{r, \Lambda}(f) k . \Pi_{r, \Lambda}\left(\mu_{s}\right) \cdot \zeta_{l} & =\left\langle\mu_{s}, \phi^{r, \Lambda, l}\right\rangle k \cdot \Pi_{r, \Lambda}(f) \cdot \zeta_{l} \\
k . \Pi_{r, \Lambda}\left(\partial_{s}^{j}\left(f * \mu_{s}\right)\right) . \zeta_{l} & =\partial_{s}^{j} \cdot\left\langle\mu_{s}, \phi^{r, \Lambda, l}\right\rangle k \cdot \Pi_{r, \Lambda}(f) . \zeta_{l} .
\end{aligned}
$$

This last equality, with (9) and Fubini's equality, yield to:

$$
\left\|S^{j}(f)\right\|^{2}=\int \sum_{l \in \mathbb{N}^{\prime}} \int_{s=0}^{\infty}\left|\partial_{s}^{j} \cdot\left\langle\mu_{s}, \phi^{r, \Lambda, l}\right\rangle\right|^{2} s^{2 j-1} d s\left|k . \Pi_{r, \Lambda}(f) . \zeta\right|^{2} d m(r, \Lambda, k) .
$$

Because of definition of $\hat{S}^{j}$, we deduce equality (8). Thus Proposition 11 is proved.

\subsection{Boundedness of $\hat{S}^{j}$}

Here, we prove Proposition 12. The tools are some computations and properties of special functions (which are recalled in Subsection 4.2). We remark:

Lemma 13. Let $f$ be a smooth function on R. For $h=1,2, \ldots$, the derivative $g^{(h)}(s)$ of $g(s)=f\left(s^{2}\right)$ is a linear combination of $s^{d(j, h)} f^{\left(h^{\prime}+j\right)}\left(s^{2}\right)$, where:

$$
\left\{\begin{array}{l}
\text { if } h=2 h^{\prime}, d(j, h)=2 j, \text { on } 0 \leq j \leq h^{\prime}, \\
\text { if } h=2 h^{\prime}-1, d(j, h)=2 j+1, \text { on } 0 \leq j \leq h^{\prime}-1 .
\end{array}\right.
$$

We remark $d(j, h)+h=2 h^{\prime}+2 j$.

We use also lemmas, (whose main lines of proof are given in the following subsection), and the following notations: we fix $\phi=\phi^{r, \Lambda, l} \in \mathscr{P}$ and $h=$ $1,2, \ldots ;$ we set for $n, \tilde{h} \in \mathrm{N}$ :

$$
\check{b}^{\tilde{h}, n}(t, s)=\int_{S_{1}^{(v)}}\left(\text { irtx }_{v}\right)^{\tilde{h}} e^{i t s r x_{v}} \partial_{s^{\prime}}^{n}\left[\prod_{j=1}^{v^{\prime}} \mathscr{L}_{l_{j}}\left(\lambda_{j} s^{\prime} r^{2} \frac{\left|\mathrm{pr}_{j}(X)\right|^{2}}{2}\right)\right]_{s^{\prime}=s^{2}} d \sigma_{v}(X) .
$$

We denote for $g=\left(h_{1}, h_{2}\right) \in \mathbf{N}^{2}, j=\left(j_{1}, j_{2}\right) \in \mathbf{N}^{2}$ :

$$
I^{g, j}:=\int_{0}^{\infty}\left|b^{g, j}(s)\right|^{2} s^{2 h-1} d s,
$$




$$
\begin{gathered}
b^{g, j}(s):=s^{d_{1}+d_{2}} \int_{r=0}^{1} \check{b}^{\tilde{h}, h_{1}^{\prime}+j_{1}}(t, s) \quad \mathscr{J}_{\frac{z-2}{2}}^{\left(h_{2}^{\prime}+j_{2}\right)}\left(s^{2} \sqrt{1-t^{4}}|A|\right) \\
\left(\sqrt{1-t^{4}}|A|\right)^{h_{2}^{\prime}+j_{2}} t^{v-1}\left(1-t^{4}\right)^{\frac{z-2}{2}} d t,
\end{gathered}
$$

where $\tilde{h}=h-h_{1}-h_{2}$ and for $i=1,2: d_{i}:=d\left(j_{i}, h_{i}\right)$. If $v$ is odd, we denote $\bar{h}=h_{1}+h_{2}$.

LEMMA 14. $\partial_{s}^{h}\left\langle\mu_{s}, \phi\right\rangle$ is a linear combination of $b^{g, j}(s)$ over $g, j$ such that $h_{1}+h_{2}+\tilde{h}=h$, where $\tilde{h}=0$ if $v$ is even, and $0 \leq j_{i} \leq h_{i} / 2, i=1,2$.

Proposition 12 will be implied by:

Proposition 15. The integrals $I^{g, j}$ are bounded independently of $(\Lambda, r, l)$ $\in \mathscr{P}$, for all parameters $g$, $j$ given in Lemma 14 , as long as $1 \leq h<(z-2) / 2$ (if $v \geq 4$ ).

The rest of this subsection is devoted to its proof, first if $v=2 v^{\prime}+1$ and $\bar{h} \neq 0$, or if $v=2 v^{\prime}$, then if $v=2 v^{\prime}+1$ and $\bar{h}=0$.

We will use the following property of the Bessel functions, deduced from the analyticity of the Bessel function $\mathscr{J}_{\alpha}$, its boundedness on R, and the classical majorations for $J_{\alpha}$ :

$$
\forall-1<\beta<2 \alpha \quad \forall n \in \mathrm{N}: \quad \int_{s=0}^{\infty}\left|\mathscr{J}_{\alpha}^{(n)}(s)\right|^{2} s^{\beta} d s<\infty .
$$

5.2.1. Boundedness of $I^{\left(h_{1}, h_{2}\right),\left(j_{1}, j_{2}\right)}$ if $\bar{h} \neq 0$ or $v=2 v^{\prime}$. Here, we assume $\left(\bar{h} \neq 0, v=2 v^{\prime}+1\right)$ or $\left(v=2 v^{\prime}\right)$ and we show Proposition 15 for these cases as long as $h<(z-2) / 2$. We will use majorations of $\breve{b}^{\tilde{h}, n}(t, s)$, given in the following lemma which will be proved in next subsection.

LeMma 16. The expressions $\breve{b}^{\tilde{h}, n}(t, s)$, for $0 \leq n \leq \tilde{h}$, are bounded up to a constant of $v, \tilde{h}$ by: $\left(|A| t^{2}\right)^{n} s^{-\tilde{h}} \sum_{0 \leq i \leq \tilde{h}}\left(|A| s^{2} t^{2}\right)^{i}$; if $v=2 v^{\prime}$, we assume $\tilde{h}=i=0$.

Using this lemma and Cauchy-Schwarz inequality, we obtain that $\left|b^{g, j}(s)\right|^{2}$ is then bounded up to a constant by:

$$
\begin{gathered}
|A|^{2\left(h_{2}^{\prime}+j_{2}+h_{1}^{\prime}+j_{1}+i\right)} s^{2\left(d_{1}+d_{2}\right)-2 \tilde{h}+4 i} \int_{0}^{1} t^{2\left(v-1+2 i+2\left(h_{1}^{\prime}+j_{1}\right)\right)}\left(1-t^{4}\right)^{-\frac{1}{2}} d t \\
\int_{0}^{1}\left|\mathscr{J}_{\frac{z-2}{2}}^{\left(h_{2}^{\prime}+j_{2}\right)}\left(s^{2} \sqrt{1-t^{4}}|A|\right)\left(1-t^{4}\right)^{\frac{z-2}{2}+\frac{h_{2}^{\prime}+j_{2}}{2}+\frac{1}{4}}\right|^{2} d t .
\end{gathered}
$$


Finally, $I^{g, j}$ is bounded up to a constant of $v, h$ by the maximum over $0 \leq i \leq \tilde{h}$ of:

$$
\begin{aligned}
J^{(i)}:=|A|^{2\left(h_{2}^{\prime}+j_{2}+h_{1}^{\prime}+j_{1}+i\right)} \int_{0}^{\infty} s^{2\left(d_{1}+d_{2}\right)-2 \tilde{h}+4 i} \\
\quad \int_{0}^{1}\left|\mathscr{F}_{\frac{z-2}{2}}^{\left(h_{2}^{\prime}+j_{2}\right)}\left(s^{2} \sqrt{1-t^{4}}|A|\right)\left(1-t^{4}\right)^{\frac{z-2}{2}+\frac{h_{2}^{\prime}+j_{2}}{2}+\frac{1}{4}}\right|^{2} d t s^{2 h-1} d s .
\end{aligned}
$$

We compute another expression of the exponent of $s$ (see Lemma 13) and the change of variable $s^{\prime}=s^{2} \sqrt{1-t^{4}}|A|$ :

$$
\begin{aligned}
J^{(i)=} \int_{s^{\prime}=0}^{\infty}\left|\mathscr{J}_{\frac{z-2}{2}}^{\left(h_{2}^{\prime}+j_{2}\right)}\left(s^{\prime}\right)\right|^{2} s^{\prime h_{2}^{\prime}+j_{2}+h_{1}^{\prime}+j_{1}+2 i-1} \frac{d s^{\prime}}{2} & \\
& \int_{0}^{1}\left(1-t^{4}\right)^{z-2+\frac{1}{2}-\left(h_{1}^{\prime}+j_{1}+2 i\right)} d t .
\end{aligned}
$$

Because of (10), we deduce that $J^{(i)}, i=0, \ldots, \tilde{h}, 0 \leq j_{1} \leq h_{1}^{\prime}, 0 \leq j_{2} \leq h_{2}^{\prime}$, are finite as long as $h_{1}^{\prime}+h_{2}^{\prime} \neq 0$ and $h<(z-2) / 2$. Proposition 15 is thus proved if $\left(\bar{h} \neq 0, v=2 v^{\prime}+1\right)$ or $\left(v=2 v^{\prime}\right)$.

5.2.2. Boundedness of $I^{0,0}$ if $v=2 v^{\prime}+1$ and $h=\tilde{h}$. . In this sub-subsection, we will assume $v=2 v^{\prime}+1$ and $h=\tilde{h}<(z-1) / 2$, so $r \neq 0$ and $g=j=$ $(0,0)=0$; we show Proposition 15 for this case.

We will use the following majoration of $\left|\check{b}^{h, 0}(r, s)\right|$ :

Lemma 17. If $v=2 v^{\prime}+1, \breve{b}^{h, 0}(t, s)$ is bounded up to a constant by:

$$
s^{-h} \sum_{0 \leq i \leq h}\left(|A| s^{2} t^{2}\right)^{i}
$$

Because of the previous majoration of $\breve{b}^{h, 0}(r, s)$ of Lemma $17,\left|b^{0,0}(s)\right|^{2}$ is bounded up to a constant by the maximum over $i=0, \ldots, h$ of:

$$
\begin{aligned}
& \left(\int_{0}^{1} s^{-h}\left(|A| s^{2} t^{2}\right)^{i}\left|\mathscr{J}_{\frac{z-2}{2}}\left(s^{2} \sqrt{1-t^{4}}|A|\right)\right| t^{v-1}\left(1-t^{4}\right)^{\frac{z-2}{2}} d t\right)^{2} \\
& \quad \leq \int_{0}^{1}\left|s^{-h}\left(|A| s^{2} t^{2}\right)^{i}\right| \mathscr{J}_{\frac{z-2}{2}}\left(s^{2} \sqrt{1-t^{4}}|A|\right)\left|\left(1-t^{4}\right)^{\frac{z-2}{2}}\right|^{2} d t \int_{0}^{1}\left|t^{v-1}\right|^{2} d t,
\end{aligned}
$$


by Hölder's inequality. Thus by Fubini's equality, $I^{0,0}$ is bounded up to a constant by:

$$
\begin{aligned}
\int_{0}^{1} \int_{0}^{\infty}\left(|A| s^{2} t^{2}\right)^{2 i}\left|\mathscr{J}_{\frac{z-2}{2}}\left(s^{2} \sqrt{1-t^{4}}|A|\right)\right|^{2} \frac{d s}{s}\left(1-t^{4}\right)^{z-2} d t \\
=\int_{0}^{\infty} s^{\prime 2 i}\left|\mathscr{J}_{\frac{z-2}{2}}\left(s^{\prime}\right)\right|^{2} \frac{d s^{\prime}}{2 s^{\prime}} \int_{0}^{1} t^{2 i}\left(1-t^{4}\right)^{z-2-i} d t,
\end{aligned}
$$

with Fubini's equality after the change of variable $s^{\prime}=s^{2} \sqrt{1-t^{4}}|A|$. Thus by Fubini's equality, $I^{0,0}$ is finite as long as $z-2-h>-1$ (because of the $d t$ integral) and $2 h-1<z-2$ (because of the $d s$-integral and majoration (10)).

As the other cases where proved in the previous sub-subsection, Proposition 15 is also proved as long as Lemmas 14, 16, 17, are shown.

\subsection{Proof of the technical lemmas}

In this subsection, we give the main lines of the proofs for lemmas used in the previous subsection.

5.3.1. Proof of Lemma 14. To prove Lemma 14, we need the following property of the Bessel functions:

$$
\int_{S^{n}} e^{i\langle x, y\rangle} d \sigma_{n}(y)=\mathscr{J}_{\frac{n-2}{2}}(|x|),
$$

where $\langle$,$\rangle denotes the Euclidean scalar product of \mathbf{R}^{n}$, and $|$.$| its norms.$

Let $\phi=\phi^{r, \Lambda, l} \in \mathscr{P}$. First, we will need the expression of $\left\langle\mu_{s}, \phi\right\rangle$. As the probability measure $\mu_{s}$ is radial, and with the expression of $\phi$ given in Subsection 4.2, we have:

$$
\begin{aligned}
& \left\langle\mu_{s}, \phi\right\rangle=\left\langle\mu_{s}, \Theta^{r, \Lambda, l}\right\rangle=\int_{S_{1}} \Theta^{r, \Lambda, l}(s . n) d \mu(n) \\
& =2 \int_{0}^{1} \int_{S^{v}} \int_{S^{z}} \Theta^{r, \Lambda, l}\left(s t X, s^{2} \sqrt{\left(1-t^{4}\right)} A\right) d \sigma_{z}(A) d \sigma_{v}(X) t^{v-1}\left(1-t^{4}\right)^{\frac{z-2}{2}} d t,
\end{aligned}
$$

because of the expression (2) of $\mu$. We notice:

$$
\begin{aligned}
\int_{\mathbf{S}^{z}} \Theta^{r, \Lambda, l}\left(t_{1} X, t_{2} A\right) & d \sigma_{z}(A) \\
=e^{i t_{1} r x_{v}} & \prod_{j=1}^{v^{\prime}} \mathscr{L}_{l_{j}}\left(\frac{\lambda_{j}}{2}\left|\operatorname{pr}_{j}\left(t_{1} X\right)\right|^{2}\right) \int_{\mathbf{S}^{z}} e^{i t_{2}\langle A, A\rangle} d \sigma_{z}(A) ;
\end{aligned}
$$


The last integral against $\sigma_{z}$ equals $\mathscr{J}_{\frac{z-2}{2}}\left(t_{2}|A|\right)$, because of (11). We then obtain:

$$
\begin{aligned}
\left\langle\mu_{s}, \phi\right\rangle=2 \int_{0}^{1} \int_{S^{v}} e^{i t s r x_{v}} \prod_{j=1}^{v^{\prime}} \mathscr{L}_{l_{j}}\left(\lambda_{j} s^{2} \frac{\left|\operatorname{pr}_{j}(X)\right|^{2}}{2}\right) d \sigma_{v}(X) \\
\mathscr{J}_{\frac{z-2}{2}}\left(s^{2} \sqrt{1-t^{4}}|A|\right) t^{v-1}\left(1-t^{4}\right)^{\frac{z-2}{2}} d t .
\end{aligned}
$$

We can now show Lemma 14. The expression

$$
\partial_{s}^{h}\left[e^{i r s t x_{v}} \prod_{j=1}^{v^{\prime}} \mathscr{L}_{l_{j}}\left(\lambda_{j} s^{2} t^{2} \frac{\left|\mathrm{pr}_{j}(X)\right|^{2}}{2}\right) \mathscr{J}_{\frac{z-2}{2}}\left(s^{2} \sqrt{1-t^{4}}|A|\right)\right],
$$

is a linear combination over $\tilde{h}+\bar{h}=h$ and $h_{1}+h_{2}=\bar{h}$ of:

$$
\partial_{s}^{\tilde{h}}\left[e^{i t s r x_{v}>}\right] \partial_{s}^{h_{1}}\left[\prod_{j=1}^{v^{\prime}} \mathscr{L}_{l_{j}}\left(\lambda_{j} s^{2} t^{2} \frac{\left|\operatorname{pr}_{j}(X)\right|^{2}}{2}\right)\right] \partial_{s}^{h_{2}}\left[\mathscr{J}_{\frac{z-2}{2}}\left(s^{2} \sqrt{1-t^{4}}|A|\right)\right] .
$$

Computing each derivative (using Lemma 13 for the second and the third one), Lemma 14 is proved.

5.3.2. Proof of Lemma 16 if $v=2 v^{\prime}$. To prove Lemmas 16 and 17, we will use the following remark, deduced from the properties of Laguerre functions [18, Lemma 1.5.3]:

Lemma 18. The functions $\mathscr{L}_{k}$ and its derivatives are bounded on $\mathrm{R}^{+}$, independently of $k$ (but not of the derivation order).

We assume here $v=2 v^{\prime}$. From Lemma 18, we deduce that

$$
\partial_{s^{\prime}}^{n}\left[\prod_{j=1}^{v^{\prime}} \mathscr{L}_{l_{j}}\left(\lambda_{j} s^{\prime} r^{2} \frac{\left|\mathrm{pr}_{j}(X)\right|^{2}}{2}\right)\right],
$$

is majorized up to a constant of $n, v$ by the sum of

$$
|A|^{n} \prod_{j \in J}\left(t^{2} \frac{\left|\operatorname{pr}_{j}(X)\right|^{2}}{2}\right)^{e_{j}}=\left(t^{2}|A|\right)^{n} \prod_{j \in J}\left(\frac{\left|\operatorname{pr}_{j}(X)\right|^{2}}{2}\right)^{e_{j}},
$$

where the sum runs over the subsets $J$ of $\mathrm{N} \cap\left[1, v^{\prime}\right]$, and $e_{j} \in \mathrm{N}, j \in J$, such that $\sum_{j \in J} e_{j}=n$. We thus obtain that $\breve{b}^{0, n}(t, s)$ is bounded up to a constant of $n, v$ by $\left(t^{2}|A|\right)^{n}$. Lemma 16 is then proved for $v=2 v^{\prime}$.

5.3.3. Proof of Lemma 16 if $\bar{h} \neq 0$ and of Lemma 17. We assume here $v=2 v^{\prime}+1$ and $\bar{h} \neq 0$. In this case, proof of Lemma 16 is based on suitable $\tilde{h}$ integrations by parts. We only prove the case $(\tilde{h}, n)=(1,0)$. The general case is similar. 
Let choose as atlas of the Euclidean sphere $\mathbf{S}^{v}$ of $\mathbf{R}^{v}$ two caps with poles $X_{1}$ and $-X_{1}$ :

$$
C_{1}:=\left\{X \in \mathrm{S}^{v},\left\langle X, X_{1}\right\rangle>-\frac{1}{2}\right\},
$$

and

$$
C_{2}:=\left\{X \in \mathrm{S}^{v},\left\langle X,-X_{1}\right\rangle>\frac{1}{2}\right\} .
$$

Then we fix a partition of unity, i.e. two smooth functions $\psi_{1}, \psi_{2}$ on $\mathbf{S}^{v}$ such that:

$$
\operatorname{supp} \psi_{i} \subset C_{i} \quad \text { and } \quad 0 \leq \psi_{i} \leq 1, i=1,2, \quad \text { and } \quad \psi_{1}+\psi_{2}=1 \quad \text { on } \mathbf{S}^{v} .
$$

As chart over $C_{1}$ (respectively $C_{2}$ ), we consider the stereo-graphic projection with pole $X_{1}$ (respectively $-X_{1}$ ), and we denote $C_{1}^{\prime}$ (respectively $C_{2}^{\prime}$ ) its range over $\mathrm{R}^{v-1}$. $C_{1}^{\prime}$ et $C_{2}^{\prime}$ are compact subsets of $\mathrm{R}^{v-1}$. The points $X=\sum_{i} x_{i} X_{i}$ of the sphere $\mathrm{S}^{v}$ are parameterized by the coordinates $x_{i}, i \neq 1$; furthermore, the measure $\sigma_{v}$ is mapped over $C_{i}, i=1,2$ a measure with a smooth density $D_{i}, i=1,2$ over the Lebesgue measure $d x$.

We decompose the integral $\check{b}^{1,0}(t, s)$ on this atlas and we integrate by parts. We obtain:

$$
\begin{aligned}
& \left|\breve{b}^{1,0}(t, s)\right| \\
& \quad=\left|\sum_{i=1,2} \int_{C_{i}^{\prime}} \frac{1}{s} e^{i s r t x_{v}} \partial_{x_{v}}\left[\prod_{j=1}^{v^{\prime}} \mathscr{L}_{l_{j}}\left(\lambda_{j} s^{2} t^{2} \frac{\left|\mathrm{pr}_{j}(X)\right|^{2}}{2}\right) x_{v} \psi_{i}(X) D_{i}\right] d x\right| \\
& \quad \leq \frac{1}{s} \sum_{i=1,2} \int_{C_{i}^{\prime}}\left|\partial_{x_{v}}\left[\prod_{j=1}^{v^{\prime}} \mathscr{L}_{l_{j}}\left(\lambda_{j} s^{2} t^{2} \frac{\left|\mathrm{pr}_{j}(X)\right|^{2}}{2}\right) x_{v} \psi_{i}(X) D_{i}\right]\right| d x .
\end{aligned}
$$

( $X$ is parameterized by $x$ on each $C_{i}$.) From Lemma 18, and the properties of the chosen atlas, and with $|A| \sim \sum_{j_{0}} \lambda_{j_{0}}$, we obtain the majoration of Lemma 16 for $(\tilde{h}, n)=(1,0)$. The rest of the proofs of Lemmas 16 and 17 are similar.

\section{REFERENCES}

1. Bourgain, J., Averages in the plane over convex curves and maximal operators, J. Analyse Math. 47 (1986), 69-85.

2. Coifman, Ronald R., and Weiss, Guido, Analyse harmonique non-commutative sur certains espaces homogènes, Étude de certaines intégrales singulières, Lecture Notes in Math. 242.

3. Cowling, Michael G., On Littlewood-Paley-Stein theory, in Proceedings of the Seminar on Harmonic Analysis (Pisa, 1980), number suppl. 1, pp. 21-55, 1981. 
4. Faraut, Jacques, and Harzallah, Khélifa, Deux cours d'analyse harmonique, Prog. Math. 69 (1987), Papers from the Tunis summer school held in Tunis, August 27-September 15, 1984.

5. Fischer, Véronique, Étude de deux classes de groupes nilpotents de pas, $\mathrm{PhD}$ thesis, Université d'Orsay, July 2004.

6. Folland, G. B., and Stein, Elias M., Hardy spaces on homogeneous groups, Math. Notes 28 (1982).

7. Jacobson, Nathan, Lie Algebras, Interscience Tracts in Pure and Applied Mathematics, No. 10. Interscience Publishers (a division of John Wiley \& Sons), New York-London, 1962.

8. Margulis, G. A., Nevo, A., and Stein, E. M., Analogs of Wiener's ergodic theorems for semisimple Lie groups, II, Duke Math. J. 103(2) (2000), 233-259.

9. Müller, Detlef, and Seeger, A., Singular spherical maximal operators on a class of step two nilpotent Lie groups, Israel J. Math. 141 (2004), 315-340.

10. Narayanan, E. K., and Thangavelu, S., An optimal theorem for the spherical maximal operator on the Heisenberg group, Israel J. Math. 144 (2004), 211-219.

11. Nevo, Amos, and Thangavelu, Sundaram, Pointwise ergodic theorems for radial averages on the Heisenberg group, Adv. Math. 127(2) (1997), 307-334.

12. Schmidt, Oliver, Maximaloperatoren zu hyperflächen in gruppen vom homogenen typ, Diplomarbeit an der Christian-Albrechts-Universität zu Kiel, Mai 1998.

13. Stein, Elias M., Topics in Harmonic Analysis related to the Littlewood-Paley Theory, Ann. of Math. Stud. 63 (1970).

14. Stein, Elias M., Maximal functions, I. Spherical means, Proc. Nat. Acad. Sci. U.S.A., 73 (1976), 2174-2175.

15. Stein, Elias M., Harmonic Analysis: Real-Variable Methods, Orthogonality, and Oscillatory Integrals, Princeton Math. Ser. 43 (1993).

16. Stein, Elias M., and Wainger, Stephen, Problems in harmonic analysis related to curvature, Bull. Amer. Math. Soc. 84(6) (1978), 1239-1295.

17. Szegő, Gábor, Orthogonal Polynomials, 4. ed., Amer. Math. Soc. Coll. Publ. XXIII (1975).

18. Thangavelu, Sundaram, Lectures on Hermite and Laguerre Expansions, with a preface by Robert S. Strichartz, Math. Notes 42 (1993).

19. Thangavelu, Sundaram, Harmonic analysis on the Heisenberg Group, Prog. Math. 159 (1998).

20. Titchmarsh, E. C., The Theory of Functions, Oxford University Press, 2nd ed., London, 1975.

DEPARTEMENT OF MATHEMATICS

GÖTEBORG UNIVERSITY

S-412 96 GÖTEBORG

SWEDEN

E-mail: veronfi@math.chalmers.se,veronique.fischer@math.u-psud.fr 